\title{
A review of innate immunity of silkworm, Bombyx mori
}

\author{
Jing Wan, Xiangyang Zhou* and Xiujin Zhou \\ Zhoushan Entry-Exit Inspection and Quarantine Bureau, Zhoushan 316000, China.
}

Accepted 8 February, 2012

\begin{abstract}
Bombyx mori is an important economic insect for silk production. However, many kinds of pathogens cause serious economic loss every year during sericulture. B. mori lack an acquired immune system but have a sophisticated innate immune system against foreign microorganisms. The innate immunity system of $B$. mori is composed of humoral and cellular immune reactions, including production of antimicrobial peptides (AMPs), phenoloxidase, phagocytosis, nodule formation, encapsulation and melanization. Three immunity pathways had been reported in the $B$. mori to control the gene expression and defending invading microorganisms: Toll pathway, Imd pathway and JAK-STAT pathway. It has been shown that gene families associated with recognition, modulation and effectors are conserved from vertebrates to invertebrates. Recent accomplishment in molecular immunology of $B$. mori has been reviewed in this paper. The development of zinc-finger nuclease technique and silkworm genome information is expected to accelerate silkworm immunity studies.
\end{abstract}

Key words: Bombyx mori, innate immunity, signal pathway, pattern recognition receptors.

\section{INTRODUCTION}

Insects possess an efficient and potent innate immune system to discriminate and eliminate invading pathogens and parasites, but lack acquired immunity or immunological memory similar to that present in vertebrates (Lemaitre and Hoffmann, 2007). Drosophila melanogaster, the type model of insects, has been particularly extensively studied in terms of immunity ( $\mathrm{Pal}$ and $\mathrm{Wu}, 2009)$. However, the recognition and elimination systems of insects may varied, thus the data from $D$. melanogaster may not always be applicable to other insects.

The silkworm, Bombyx mori, domesticated more than 5, 000 years ago, is an important economic insect for silk production, and is also a good model lepidopteran (Goldsmith et al., 2005). In many rural areas of China, India, Brazil and so on, sericulture is one of the main sources of income for farmers. Every year, severe economic losses happen in the sericulture industry, mainly due to the variety pathogens (Pandiarajan et al., 2011) he innate immune system of $B$. mori is also divided into two major reaction types: humoral and cellular immune responses. The first-line defense against microbes consists of structural barriers that include the epidermis, the midgut peritrophic membrane and tracheal respiratory organs and then in the hemocoele. Once in the hemocoele, the infectious microorganisms are fought by humoral and cellular responses (Tanaka et al., 2008). However, silkworm immunity studies, particularly at the molecular level, are not as advanced as those on the common vinegar fly and mosquitoes. Although some progress related to innate immunity of Bombyx mori has been published recently using silkworm genome information and modern technology, very little is known about the mechanism of innate immunity (Tian et al., 2010). So the comprehensive understanding the mechanism of the $B$. mori immunity will give us more 
information on the control of pathogens, such as virus, bacteria and fungi. Understanding the interaction between host and pathogen will help us to know the insect pathological and defense mechanism, also to exploit new biological insecticides for agriculture pest. The purpose of this review is to summarize the current knowledge on $B$. mori innate immunity, focusing on the results of recent studies, which are intended to gather as much as possible the information about the immunology of $B$. mori.

\section{HUMORAL AND CELLULAR RESPONSES TO INFECTION IN B. mori}

There are at least five types of hemocytes in the hemolymph of silkworm larvae, which can be distinguished morphologically: prohemocytes, plasmatocytes, granular cells, spherule cells and oenocytoides. The celluar responses include phagocytosis, nodule formation and encapsulation by host cells. In invertebrates, in addition to lots of enzyme cascades, a variety of agglutinin-lectins and reactive oxygen producing and phagocytic systems cooperate with immune reactions to kill invading pathogens (Bogdan et al., 2000). Invaders are detected by the defense systems, then engulfed by phagocytes, such as macrophage-like, neutrophillike and dendritic cell, and then foreign invaders are internalized as phagosomes and finally killed (Greenberg and Grinstein, 2002).

The humoral responses include the activation of cascades of constitutive proteins present in the hemolymph, such as those in the prophenoloxidase cascade, leading to melanization (deposition of melanin pigments onto pathogens and the wounded sites) and the coagulation cascade, and the activation of intracellular signaling pathways that produce defense proteins such as anti-microbial peptides in the immune responsive tissues and cells. The antimicrobial peptide (AMPs) genes are induced by microbial challenge in the fat body (equivalent of the mammalian liver), followed by the secretion of these peptides into the haemolymph, which is the hallmark of the humoral reactions (Hoffmann and Reichhart, 2002). These antimicrobial peptides from several families reach high concentrations in the hemolymph and efficiently kill invading microorganisms (Ferrandon et al., 2007), in the midgut of insect, the reactive intermediates of oxygen or nitrogen play a important role in killing the invaders (Bogdan et al., 2000; Nappi and Vass, 2001). In holometabolous insects, such as B. mori, AMP gene transcription is inducible. Absent from uninfected insects, AMPs are rapidly produced upon infection by the fat body or by epithelia such as the gut or the trachea. The humoral and cellular responses are not easy to divide, as many humoral factors affect hemocyte function and hemocytes are an important source of many humoral molecules. There also is considerable overlap between humoral and cellular defenses in processes like the recognition of foreign intruders.

\section{SIGNAL PATHWAY IN THE HOST DEFENSE OF $B$. mori}

There are two immunity pathways in the body to control the gene expression, defending invading microorganisms. The Toll pathway is activated primarily in response to fungal and some Gram-positive bacterial infections, whereas the Imd pathway is activated predominantly in response to Gram-positive and other Gram-negative bacterial infections.

It is well known that Toll activation during the immune responses is strictly dependent on the product of the Spaetzle gene. This Spaetzle protein is a cystine-knot molecule with structural similarities to mammalian neurotrophins, and requires proteolytic cleavage for full biological activity (Mizuguchi et al., 1998). This cleavage is induced by a proteolytic cascade activated as an early result of infection. Toll receptors, which are characterized by the extracellular leucine rich repeat (LRR) arrays and an intracellular Toll-interleukin-1 receptor (TIR) domain, play important roles in innate immunity in invertebrates and adaptive immunity in vertebrate animals (Taylor et al., 2008).

There have three partners interacted with the intracytoplasmic TIR domain of Toll, each of them has a death domain. Two of these are adaptor proteins: the Drosophila homologue of MyD88 (Horng and Medzhitov, 2001; Sun et al., 2004; Tauszig-Delamasure et al., 2002), which in addition to the death domain has a TIR domain similar to that of Toll with which it associates, and tube. Tube has no obvious mammalian homologue. The third death domain protein in this receptor-adaptor complex is Pelle, which has a serine-threonine kinase domain and is homologous to mammalian IRAKs (interleukin-1 receptorassociated kinases) (Janssens and Beyaert, 2003). The TIR [Toll-inter- leukin-1(IL-1)] receptor domain of Toll binds to the TIR domain of MyD88, however, binding occurs only when the receptor is active (Horng and Medzhitov, 2001; Sun et al., 2004; Tauszig-Delamasure et al., 2002). Subsequently, through interactions of death domains, assembly of the signaling complex containing MyD88, Tube and Pelle occurs (Sun et al., 2004).The increased local concentration of Pelle might lead to transphosphorylation and stimulation of the Pelle kinase activity (Shen and Manley, 2002). The activated Pelle, in an as yet undefined manner, acts on the cytoplasmic Dorsal-Cactus and Dif-Cactus complexes. Dif and Dorsal are NF-kB homologues and are normally retained in the cytoplasm by the IkB-related inhibitor Cactus (Brennan and Anderson, 2004; Hoffmann, 2003; Hultmark, 2003). After signal-induced degradation of Cactus, Dif and Dorsal translocate to the nucleus and activate the expression of antimicrobial peptide genes.

The Imd pathway controls the expression of many antimicrobial peptide genes in response to Gram-negative bacterial infection (Brennan and Anderson, 2004; Hoffmann, 2003; Hultmark, 2003; Lemaitre, 2004).

Activation of this pathway triggers a cascade of kinases 
(DmTAK, IKK), which ultimately phosphorylates the Rel protein Relish (Leulier et al., 2002; Naitza et al., 2002). Phosphorylated Relish is then cleaved by the DREDD caspase dissociating the I-B like domain from the Rel DNA binding domain, which can then translocate into the nucleus (Leulier et al., 2000; Stoven et al., 2003). The pattern-recognition receptor for the Imd pathway appears to be PGRP-LC, which contains a putative transmembrane domain (Choe et al., 2002; Gottar et al., 2002). There are two main families of pattern-recognition receptors, the peptidoglycan-recognition proteins (PGRPs) and the Gram-negative binding proteins (GNPBs), which have homology to enzymes (amidase or glucanase), can bind to molecules (lipopolysaccharides, peptidoglycans and glucans) associated with microbes, then trigger signaling cascades to activated immune cells or the transcription of AMPs to isolate or kill invaders.

There have another pathway which had been reported in the response of insect immunity, that is the JAK-STAT pathway, like the Toll pathway, the JAK-STAT pathway also plays dual biological function in development and immunity. The STAT protein is accumulated in the nucleus after immune challenge in the mosquito, it was first suggested that the JAK-STAT pathway was involved in insect immunity (Barillas-Mury et al., 1999). In Drosophila, the JAK-STAT pathway plays an important role in hematopoiesis, stress responses, stem cell proliferation, and antiviral immunity, but its role in the defense against natural bacterial pathogens is unknown (Agaisse and Perrimon, 2004; Lee, 2009; Singh et al., 2007). The silkworm were orally infected by Staphylococcus aureus and Escherichia coli, all the three JAK/STAT pathway genes, such as Dome, Hop and Stat1 were significantly up-regulated (Wu et al., 2010). The results showed that JAK-STAT pathway also participates in the antimicrobial response of the insect gut, which is consistent with the previous study results by the Buchon group (Buchon et al., 2009).

\section{PATTERN RECOGNITION RECEPTORS IN THE INNATE IMMUNE SYSTEM}

Invertebrate innate immune reactions are triggered when so-called pathogen-associated molecular patterns (PAMPs) of microorganisms are detected and recognized as "non-self" by the pattern recognition receptors (PRRs) of the host. So far, seven groups of PRRs have been identified in invertebrates, including peptidoglycanrecognition proteins (PGRP), thioester-containing proteins (TEP), Gram-negative binding proteins (GNBP), multidomain scavenger receptors (SCR), C-type lectins(CTL), galactoside-binding lectin (galectin) and fibrinogen- like domain immunolectin.

\section{Peptidoglycan recognition proteins (PGRPs)}

PGRPs have been shown to play a central role in the recognition of invading microorganisms in insect immunity (Hultmark, 2003; Royet et al., 2005; Steiner, 2004). Silkworm PGRP is a hemolymph protein that specifically binds peptidoglycan from bacterial cell walls. It is required for the peptidoglycan-mediated activation of the phenoloxidase cascade (Yoshida et al., 1986, 1996). There is 12 distinct PGRP genes with conserved PGRP domains in the genome of Bombyx. Six belong to the short subfamily, four of the S-type PGRPs (BmPGRP-S3, -S4, $S 5$ and $-S 6$ ) are located in tandem on chromosome No.16 and predicted to be secreted proteins, because all transcripts from these genes have putative signal peptides, BmPGRP-S1 and S2 are surmised to be secreted proteins. BmPGRP-L1, $-L 2,-L 3,-L 4$ and $-L 5$ are located in tandem within $70 \mathrm{~kb}$ on chromosome No. 1, BmPGRP-L6 possesses no signal peptide and transmembrane domain and is thought to be present in the cytoplasm. BmPGRP-L2, -L3 and -L5 appear to be secreted proteins, whereas BmPGRP-L1 and -L4 appear to be transmembrane proteins (Tanaka et al., 2008). Recent studies on the binding specificity of PGRP to PGN using human and Drosophila recombinant PGRPs and synthetic PGN fragments have demonstrated that the PGRPs distinguish between PGNs from different bacteria based on the differences in both the diamino amino acid at the third position of the stem peptide and the structures of the peptide bridge cross-linking the stems (Kumar et al., 2005; Swaminathan et al., 2006).

\section{$\beta$-Glucan recognition protein ( $\beta$ GRP)}

$\beta$ GRP contains two functionally different proteins, one of which binds to $\beta-1,3$-glucan and the other dubbed Gramnegative binding protein (GNBP) binds to Gram-negative bacteria or Gram-positive bacteria. The previous studies showed that $B$. mori has four $\beta G R P$ genes including a gene designated $B m \beta G R P 1$ encoding the first $\beta G R P$ isolated by Yoshida group (Yoshida et al., 1986), a gene designated Bm $\beta G R P 2$ encoding GNBP isolated by Lee group (Lee et al., 1996) and two novel genes designated $B m \beta G R P 3$ and 4 , all Bm $\beta G R P s$ are assumed to be secreted proteins because they have putative signal peptides (Tanaka et al., 2008). A cDNA of $\beta G R P$ was cloned from the silkworm $B$. mori, was concluded Glucanases and the current pattern-recognition proteins that contain a glucanase-like region seem to have a common origin in their molecular evolution (Ochiai and Ashida, 2000).

\section{Fibrinogen-related proteins (FREPs)}

FREPs contain FBG (fibrinogen-like domain) domains in their C-terminal region, are found universally in vertebrates and invertebrates. These proteins are known to participate in recognition of bacteria and parasites in invertebrates (Gokudan et al., 1999; Schroder et al., 2003). 
In invertebrates, FREPs are involved in cell-cell interaction, bacterial recognition, and antimicrobial responses (Gokudan et al., 1999; Schroder et al., 2003; Wang et al., 2004). There were only three FREP genes in the genome of $B$. mori, and species-specific gene expansion seen in Anopheles, Aedes, Drosophila and Tribolium was not found in Bombyx (Tanaka et al., 2008).

\section{The multidomain scavenger receptor (SCR)}

SCR family contains eight subfamilies from $A$ to $H$ depending on the amino acid sequences. SCRs recognize multiple ligands and serve to remove apoptotic cells and bacteria (Peiser et al., 2002). Four genes of encoding SRCR were detected in the genome of Bombyx, they encode BmSCRASP2 (Corin-like), BmSCRAL1 (Lox2like), BmSCRAC1 (CG3921-like) and BmSCRASP4 proteins (Tanaka et al., 2008).

\section{C-type lectins (CTLs)}

CTLs can act as pattern recognition receptors in innate immunity, which are $\mathrm{Ca}^{2+}$-dependent proteins, and outside of cells, they function as secreted proteins or membranebound proteins (Drickamer and Taylor, 1993). CTLs play an important role as recognition proteins for microorganisms in immune reactions during the early phase of microbial infection (Watanabe et al., 2006). Invertebrate CTLs are involved in immune responses including PPO activation (Yu and Kanost, 2000), hemocyte nodule formation (Koizumi et al., 1999), opsonization and microbial clearance (Jomori and Natori, 1992; Yu and Kanost, 2003). Twenty one CTL genes (BmCTL1-21) were detected in the Bombyx genome (Tanaka et al., 2008). In the lepidopteran insects, a group of C-type lectins that contain two tandem CRDs has been suggested to have PRR functions. Two dual-CRD C-type lectins were isolated from the silkworm $B$. mori, which were designated as $B$. mori LPS-binding protein (BmLBP) and $B$. mori multiple saccharide-binding protein (BmMBP). $B m L B P$ and BmMBP have distinct pathogen recognition characteristics; BmLBP binds to Gram-negative bacteria, whereas BmMBP primarily binds to Gram-positive bacteria and yeast (Koizumi et al., 1997; Watanabe et al., 2006). Three novel C-type lectins cDNAs were obtained from the $B$. mori, which were designated BmLEL-1, -2 , and -3 . They concluded that the novel C-type lectins might play a role in the innate immunity in these tissues (Takase et al., 2009).

\section{Galectin}

Galectin is a lectin that specifically binds to $\beta$-galactoside sugar and contains evolutionary conserved CRDs.
Galectin is thought to participate in microbial recognition or phagocytosis in flies and mosquitoes (Pace and Baum, 2004). There are four genes (BmGalectin1-4) encoding Galectin in the Bombyx genome (Tanaka et al., 2008).

\section{Thioester-containing protein (TEP)}

TEP is a secretory protein and related to mammalian $\alpha_{2}-$ macroglobulin. AgTEP1 (ca.160 kDa) is processed upon infection with Gram-negative bacteria and the resultant Cterminal $80 \mathrm{kDa}$ polypeptide binds to the surface of Gramnegative bacteria via the $\mathrm{y}$-glutamyl ketone group of the peptide, promoting phagocytosis by plasmatocytes (Levashina et al., 2001). Three TEP genes (BmTEP1-3) were identified in the $B$. mori genome during Bacillus bombyseptieus infection, which is unlike $A$. gambiae contains 15 TEPs (Huang et al., 2009).

\section{The immunoglobulin (lg)-superfamily}

The immunoglobulin proteins are known for their abilities to specifically recognize and adhere to cells. In mammals immune system, Igs are present on the B-lymphocyte surface and play crucial roles in transducing the signals of cytoplasmic and nuclear effectors and delivering antigens to the cellular compartment where these are degraded (Litman et al., 1999). To date, several reports have shown that IgSF members play crucial roles in invertebrate immune response. This was the first insect IgSF molecule to be considered a PRR, since it has broad specificity for lipopolysaccharide and lipotechoic acid (Daffre and Faye, 1997; Gokudan et al., 1999; Yu and Kanost, 2002) and is a major player during Gram-negative bacterial infections (Eleftherianos et al., 2006a, b; 2007). Hemolin gene is the only one player in the immunoglobulin superfamily of invertebrate, which was found only from lepidopteran insects (Bao et al., 2007). Which is induced and expressed after baculovirus and dsRNA introduction, suggesting that hemolin is used in antiviral defense (Hirai et al., 2004). The hemolin gene was cloned by our group from the fat body of $B$. mori, the result showed that the recombinant hemolin obtained from in vitro expression by using baculovirus expression system had antibacterial activity, being an effective inhibitor to the growth of Bacillus thuringiensis. Hemolin is down-regulated rather than up-regulated in most cases of infection in the silkworm fat body over the time course of the infection with the Gram-negative bacteria E. coli and also the Grampositive bacteria $B$. thuringiensis (Huang et al., 2009). It was speculated because the immune response kinetic is very sensitive. $133 \mathrm{IgSF}$ proteins were predicted in the silkworm genome, Comparison with similar proteins in other model organisms indicated that IgSF proteins are conserved but have rapidly evolved from worms to human beings (Huang et al., 2009). 
Table 1. Gene counts belonging to immunity-related gene families of Bombyx.

\begin{tabular}{lc}
\hline Gene family & B. mori \\
\hline Recognition & \\
PGRP & 11 \\
GNBP & 4 \\
Fibrinogen-related protein & 3 \\
Scavenger & 12 \\
C-type lectin & 22 \\
Galectin & 2 \\
Signaling & \\
Spätzle & 6 \\
Toll & 13 \\
CLIP-SP & 15 \\
Serpin & 15 \\
REL & 2 \\
Components\# & 17 \\
Effectors & \\
PPO & 3 \\
TEP & 6 \\
LYS & 3 \\
AMP* & 39 \\
Others & \\
Caspase & 4 \\
Catalase & 7 \\
Inhibitors of apoptosis & 5 \\
Peroxidase & 23 \\
Superoxide dismutatse & 6 \\
Total & 218 \\
\hline
\end{tabular}

\#:The components from immune pathways including Toll, Imd, JNK and JAKSTAT pathway. ": Cecropins, moricine, gloverins, attacins, enbocins, lebocins, defensin, diptericins, drosomycins, drosocin, metchnikowin, gambicin, holotricin, abaecin, apidaecin, apisimin and hymenoptaecin.

\section{DISCUSSION}

The silkworm is agriculturally very important for silk production, so their pathological and genetic studies on diseases have been very significantly and extensively carried on. Severe economic losses caused by the pathologies, such as virus, bacterium and fungus so on, so the comprehensive understanding the innate immunity pathway and host-pathogen interaction will attribute for us defending economic losses and benefit from the silk industry. In the past several years, the studies on the innate immunity of $B$. mori have gained significant results, such as much recognition, modulation, signaling, effectors and other immune molecules (Table 1).

The comparison between Toll and Imd pathway enable us to further understand the mechanisms of innate immune responses. Recent years, phylogenitic analysis that many immunity members in the invertebrate immunity have very similar defending genes to the mammals, this result illustrate the similarities between some of the strategies used both by insects and mammals to sense infection and amplify the information (Hoffmann, 2003). Even though, some paper published that the structural and function similarities between the Toll and the TLRdependent activation of NF-kB has been interpreted as evidence for the existence of a common ancestor and shared mechanisms between the vertebrate and invertebrate innate immune systems (Royet and Dziarski, 2007). Many reports revealed that stem cell is involved in the regeneration by destroy and in maintain the homeostasis in the Drosophila intestinal. So we speculated that some kinds of stem cell are also involved in the intestinal homeostasis of silkworm.

Recently, a genome-wide analysis of immune-related genes of $B$. mori revealed that the factors associated with the signal transduction pathways are conserved in $B$. mori and non-lepidopteran insects (Tanaka et al., 2008). However, the function of most genes encoding recognition proteins in B. mori isstill unknown. Nonetheless, in the near future, the immune-related genes can be elucidated by the development of functional analyses such as RNA interference (RNAi) (Fujita et al., 2009), transgenic technology, GAL4/UAS system (Kobayashi et al., 2011) and zinc-finger nuclease technique (Takasu et al., 2010). Comprehensively exploring the mechanism of innate immunity between insect will give us much information about the host and pathogen interaction, defensive mechanisms evolved in the host in response to infection and anti-defensive/immunosuppression molecules released by pathogen, all of these will give us more references about the immunity mechanisms in the mammals and understand the defense mechanism in the insect, and also which will contribute to the sericultural field by establishing transgenic non-susceptible strains of silkworm and to agriculture for better control of lepidopteran pests.

\section{REFERENCES}

Agaisse $\mathrm{H}$, Perrimon $\mathrm{N}$ (2004). The roles of JAK/STAT signaling in Drosophila immune responses. Immunol. Rev. 198:72-82.

Bao Y, Yamano Y, Morishima I (2007). Induction of hemolin gene expression by bacterial cell wall components in eri-silkworm, Samia cynthia ricini. Comp. Biochem. Physiol. B. Biochem. Mol. Biol. 146(1):147-151.

Barillas-Mury C, Han YS, Seeley D, Kafatos FC (1999). Anopheles gambiae Ag-STAT, a new insect member of the STAT family, is activated in response to bacterial infection. EMBO J. 18(4):959-967.

Bogdan C, Rollinghoff M, Diefenbach A (2000). Reactive oxygen and reactive nitrogen intermediates in innate and specific immunity. Curr. Opin. Immunol. 12(1):64-76.

Brennan CA, Anderson KV (2004). Drosophila: the genetics of innate immune recognition and response. Annu. Rev. Immunol. 22:457-483.

Buchon N, Broderick NA, Poidevin M, Pradervand S, Lemaitre B (2009). Drosophila intestinal response to bacterial infection: activation of host defense and stem cell proliferation. Cell Host Microbe. 5(2):200-211.

Choe KM, Werner T, Stoven S, Hultmark D, Anderson KV (2002). Requirement for a peptidoglycan recognition protein (PGRP) in Relish activation and antibacterial immune responses in Drosophila. Science 296(5566):359-362. 
Daffre S, Faye I (1997). Lipopolysaccharide interaction with hemolin, an insect member of the Ig-superfamily. FEBS Lett. 408(2):127-130.

Drickamer K, Taylor ME (1993). Biology of animal lectins. Annu. Rev. Cell Biol. 9:237-264.

Eleftherianos I, Gokcen F, Felfoldi G, Millichap PJ, Trenczek TE, ffrenchConstant RH, Reynolds SE (2007). The immunoglobulin family protein Hemolin mediates cellular immune responses to bacteria in the insect Manduca sexta. Cell Microbiol. 9(5):1137-1147.

Eleftherianos I, Marokhazi J, Millichap PJ, Hodgkinson AJ, Sriboonlert A, ffrench-Constant RH, Reynolds SE (2006a). Prior infection of Manduca sexta with non-pathogenic Escherichia coli elicits immunity to pathogenic Photorhabdus luminescens: roles of immune-related proteins shown by RNA interference. Insect Biochem. Mol. Biol. 36(6):517-525.

Eleftherianos I, Millichap PJ, ffrench-Constant RH, Reynolds SE (2006b). RNAi suppression of recognition protein mediated immune responses in the tobacco hornworm Manduca sexta causes increased susceptibility to the insect pathogen Photorhabdus. Dev. Comp. Immunol. 30(12):1099-1107.

Ferrandon D, Imler JL, Hetru C, Hoffmann JA (2007). The Drosophila systemic immune response: sensing and signalling during bacterial and fungal infections. Nat. Rev. Immunol. 7(11):862-874.

Fujita K, Sagisaka A, Tomimoto K, Ishibashi J, Imanishi S, Yamakawa M, Tanaka H (2009). DNA vector-based RNA interference in cell lines derived from Bombyx mori. Biosci. Biotechnol. Biochem. 73(9):20262031.

Gokudan S, Muta T, Tsuda R, Koori K, Kawahara T, Seki N, Mizunoe Y, Wai SN, Iwanaga S, Kawabata S (1999). Horseshoe crab acetyl group-recognizing lectins involved in innate immunity are structurally related to fibrinogen. Proc. Natl. Acad. Sci. U. S. A. 96(18):1008610091.

Goldsmith MR, Shimada T, Abe H (2005). The genetics and genomics of the silkworm, Bombyx mori. Annu. Rev. Entomol. 50:71-100.

Gottar M, Gobert V, Michel T, Belvin M, Duyk G, Hoffmann JA, Ferrandon D, Royet J (2002). The Drosophila immune response against Gram-negative bacteria is mediated by a peptidoglycan recognition protein. Nature 416(6881):640-644.

Greenberg S, Grinstein S (2002). Phagocytosis and innate immunity. Curr. Opin. Immunol. 14(1):136-145.

Hirai M, Terenius O, Li W, Faye I (2004). Baculovirus and dsRNA induce Hemolin, but no antibacterial activity, in Antheraea pernyi. Insect Mol. Biol. 13(4):399-405.

Hoffmann JA (2003). The immune response of Drosophila. Nature 426(6962):33-38.

Hoffmann JA, Reichhart JM (2002). Drosophila innate immunity: an evolutionary perspective. Nat. Immunol. 3(2):21-126.

Horng T, Medzhitov R (2001). Drosophila MyD88 is an adapter in the Toll signaling pathway. Proc. Natl. Acad. Sci. U. S. A. 98(22):1265412658.

Huang L, Cheng T, Xu P, Cheng D, Fang T, Xia Q (2009). A genomewide survey for host response of silkworm, Bombyx mori during pathogen Bacillus bombyseptieus infection. PLoS One 4(12):e8098.

Hultmark D (2003). Drosophila immunity: paths and patterns. Curr. Opin. Immunol. 15(1):12-19.

Janssens S, Beyaert R (2003). Functional diversity and regulation of different interleukin-1 receptor-associated kinase (IRAK) family members. Mol. Cell 11(2):293-302.

Jomori T, Natori S (1992). Function of the lipopolysaccharide-binding protein of Periplaneta americana as an opsonin. FEBS Lett. 296(3):283-286.

Kobayashi I, Kojima K, Uchino K, Sezutsu H, lizuka T, Tatematsu K, Yonemura N, Tanaka H, Yamakawa M, Ogura E, Kamachi Y, Tamura $T$ (2011). An efficient binary system for gene expression in the silkworm, Bombyx mori, using GAL4 variants. Arch. Insect Biochem. Physiol. 76(4):195-210.

Koizumi N, Imamura M, Kadotani T, Yaoi K, Iwahana H, Sato R (1999). The lipopolysaccharide-binding protein participating in hemocyte nodule formation in the silkworm Bombyx mori is a novel member of the C-type lectin superfamily with two different tandem carbohydraterecognition domains. FEBS Lett. 443(2):139-143.

Koizumi N, Morozumi A, Imamura M, Tanaka E, Iwahana H, Sato R (1997). Lipopolysaccharide-binding proteins and their involvement in the bacterial clearance from the hemolymph of the silkworm Bombyx mori. Eur. J. Biochem. 248(1):217-224.

Kumar S, Roychowdhury A, Ember B, Wang Q, Guan R, Mariuzza RA, Boons GJ (2005). Selective recognition of synthetic lysine and mesodiaminopimelic acid-type peptidoglycan fragments by human peptidoglycan recognition proteins $\mid\{a l p h a\}$ and S. J. Biol. Chem. 280(44):37005-37012.

Lee WJ (2009). Bacterial-modulated host immunity and stem cell activation for gut homeostasis. Genes Dev. 23(19):2260-2265.

Lee WJ, Lee JD, Kravchenko VV, Ulevitch RJ, Brey PT (1996). Purification and molecular cloning of an inducible gram-negative bacteria-binding protein from the silkworm, Bombyx mori. Proc. Natl. Acad. Sci. U. S. A. 93(15):7888-7893.

Lemaitre B (2004). The road to Toll. Nat. Rev. Immunol. 4(7):521-527.

Lemaitre B, Hoffmann J (2007). The host defense of Drosophila melanogaster. Annu. Rev. Immunol. 25:697-743.

Leulier F, Rodriguez A, Khush RS, Abrams JM, Lemaitre B (2000). The Drosophila caspase Dredd is required to resist gram-negative bacterial infection. EMBO Rep. 1(4):353-358.

Leulier F, Vidal S, Saigo K, Ueda R, Lemaitre B (2002). Inducible expression of double-stranded RNA reveals a role for dFADD in the regulation of the antibacterial response in Drosophila adults. Curr. Biol. 12(12):996-1000.

Levashina EA, Moita LF, Blandin S, Vriend G, Lagueux M, Kafatos FC (2001). Conserved role of a complement-like protein in phagocytosis revealed by dsRNA knockout in cultured cells of the mosquito, Anopheles gambiae. Cell 104(5):709-718.

Litman GW, Anderson MK, Rast JP (1999). Evolution of antigen binding receptors. Annu. Rev. Immunol. 17:109-147.

Mizuguchi K, Parker JS, Blundell TL, Gay NJ (1998). Getting knotted: a model for the structure and activation of Spatzle. Trends Biochem. Sci. 23(7):239-242.

Naitza S, Rosse C, Kappler C, Georgel P, Belvin M, Gubb D, Camonis J, Hoffmann JA, Reichhart JM (2002). The Drosophila immune defense against gram-negative infection requires the death protein dFADD. Immunity 17(5):575-581.

Nappi AJ, Vass E (2001). The effects of nitric oxide on the oxidations of I-dopa and dopamine mediated by tyrosinase and peroxidase. J. Biol. Chem. 276(14):11214-11222.

Ochiai M, Ashida M (2000). A pattern-recognition protein for beta-1,3glucan. The binding domain and the cDNA cloning of beta-1,3-glucan recognition protein from the silkworm, Bombyx mori. J. Biol. Chem. 275(7):4995-5002.

Pace KE, Baum LG (2004). Insect galectins: roles in immunity and development. Glycoconj. J. 19(7-9):607-614.

Pal S, Wu LP (2009). Lessons from the fly: pattern recognition in Drosophila melanogaster. Adv. Exp. Med. Biol, 653:162-174.

Pandiarajan J, Cathrin BP, Pratheep T, Krishnan M (2011). Defense role of the cocoon in the silk worm Bombyx mori L. Rapid Commun. Mass Spectrom. 25(21):3203-3206.

Peiser L, Mukhopadhyay S, Gordon S (2002). Scavenger receptors in innate immunity. Curr. Opin. Immunol. 14(1):123-128.

Royet J, Dziarski R (2007). Peptidoglycan recognition proteins: pleiotropic sensors and effectors of antimicrobial defences. Nat. Rev. Microbiol. 5(4):264-277.

Royet J, Reichhart JM, Hoffmann JA (2005). Sensing and signaling during infection in Drosophila. Curr. Opin. Immunol. 17(1):11-17.

Schroder HC, Ushijima H, Krasko A, Gamulin V, Thakur NL, Diehl-Seifert B, Muller IM, Muller WE (2003). Emergence and disappearance of an immune molecule, an antimicrobial lectin, in basal metazoa. A tachylectin-related protein in the sponge Suberites domuncula. J. Biol. Chem. 278(35):32810-32817.

Shen B, Manley JL (2002). Pelle kinase is activated by autophosphorylation during Toll signaling in Drosophila. Development 129(8):1925-1933.

Singh SR, Liu W, Hou SX (2007). The adult Drosophila malpighian tubules are maintained by multipotent stem cells. Cell Stem Cell $1(2): 191-203$.

Steiner $\mathrm{H}$ (2004). Peptidoglycan recognition proteins: on and off switches for innate immunity. Immunol. Rev. 198:83-96.

Stoven S, Silverman N, Junell A, Hedengren-Olcott M, Erturk D, Engstrom Y, Maniatis T, Hultmark D (2003). Caspase-mediated 
processing of the Drosophila NF-kappaB factor Relish. Proc. Natl. Acad. Sci. U. S. A. 100(10):5991-5996.

Sun H, Towb P, Chiem DN, Foster BA, Wasserman SA (2004). Regulated assembly of the Toll signaling complex drives Drosophila dorsoventral patterning. EMBO J. 23(1):100-110.

Swaminathan CP, Brown PH, Roychowdhury A, Wang Q, Guan R, Silverman N, Goldman WE, Boons GJ, Mariuzza RA (2006). Dual strategies for peptidoglycan discrimination by peptidoglycan recognition proteins (PGRPs). Proc. Natl. Acad. Sci. U. S. A. 103(3):684-689.

Takase H, Watanabe A, Yoshizawa Y, Kitami M, Sato R (2009). Identification and comparative analysis of three novel C-type lectins from the silkworm with functional implications in pathogen recognition. Dev. Comp. Immunol. 33(6):789-800.

Takasu Y, Kobayashi I, Beumer K, Uchino K, Sezutsu H, Sajwan S, Carroll D, Tamura T, Zurovec M (2010). Targeted mutagenesis in the silkworm Bombyx mori using zinc finger nuclease mRNA injection. Insect Biochem. Mol. Biol. 40(10):759-765.

Tanaka H, Ishibashi J, Fujita K, Nakajima Y, Sagisaka A, Tomimoto K, Suzuki N, Yoshiyama M, Kaneko Y, Iwasaki T, Sunagawa T, Yamaji $\mathrm{K}$, Asaoka A, Mita K, Yamakawa M (2008). A genome-wide analysis of genes and gene families involved in innate immunity of Bombyx mori. Insect Biochem. Mol. Biol. 38(12):1087-1110.

Tauszig-Delamasure S, Bilak H, Capovilla M, Hoffmann JA, Imler JL (2002). Drosophila MyD88 is required for the response to fungal and Gram-positive bacterial infections. Nat. Immunol. 3(1):91-97.

Taylor DL, Zhong L, Begg DJ, de Silva K, Whittington RJ (2008). Toll-like receptor genes are differentially expressed at the sites of infection during the progression of Johne's disease in outbred sheep. Vet. Immunol. Immunopathol. 124(1-2):132-151.

Tian L, Guo E, Diao Y, Zhou S, Peng Q, Cao Y, Ling E, Li S (2010). Genome-wide regulation of innate immunity by juvenile hormone and 20-hydroxyecdysone in the Bombyx fat body. BMC Genomics, 11:549.

Wang X, Rocheleau TA, Fuchs JF, Hillyer JF, Chen CC, Christensen BM (2004). A novel lectin with a fibrinogen-like domain and its potential involvement in the innate immune response of Armigeres subalbatus against bacteria. Insect Mol. Biol. 13(3):273-282.
Watanabe A, Miyazawa S, Kitami M, Tabunoki H, Ueda K, Sato R (2006). Characterization of a novel C-type lectin, Bombyx mori multibinding protein, from the $B$. mori hemolymph: mechanism of widerange microorganism recognition and role in immunity. J. Immunol. 177(7):4594-4604.

Wu S, Zhang X, He Y, Shuai J, Chen X, Ling E (2010). Expression of antimicrobial peptide genes in Bombyx mori gut modulated by oral bacterial infection and development. Dev. Comp. Immunol. 34(11):1191-1198.

Yoshida H, Kinoshita K, Ashida M (1996). Purification of a peptidoglycan recognition protein from hemolymph of the silkworm, Bombyx mori. J. Biol. Chem. 271(23):13854-13860.

Yoshida H, Ochiai M, Ashida M (1986). Beta-1,3-glucan receptor and peptidoglycan receptor are present as separate entities within insect prophenoloxidase activating system. Biochem. Biophys. Res. Commun. 141(3):1177-1184.

Yu XQ, Kanost MR (2000). Immulectin-2, a lipopolysaccharide-specific lectin from an insect, Manduca sexta, is induced in response to gramnegative bacteria. J. Biol. Chem. 275(48):37373-37381.

Yu XQ, Kanost MR (2002). Binding of hemolin to bacterial lipopolysaccharide and lipoteichoic acid. An immunoglobulin superfamily member from insects as a pattern-recognition receptor. Eur. J. Biochem. 269(7):1827-1834.

Yu XQ, Kanost MR (2003). Manduca sexta lipopolysaccharide-specific immulectin-2 protects larvae from bacterial infection. Dev. Comp. Immunol. 27(3):189-196. 